\title{
On a Graded Inclusion of Bipolar Fuzzy Relations
}

\author{
Ludovic Liétard, ${ }^{1}$ Olivier Pivert,${ }^{2}$ Daniel Rocacher ${ }^{2}$ \\ ${ }^{1}$ Irisa-IUT Lannion, France, ludovic.lietard@univ-rennes1.fr \\ ${ }^{2}$ Irisa-Enssat, Lannion, France, \{pivert, rocacher\}@enssat.fr
}

\begin{abstract}
This paper deals with database fuzzy queries in the context where the fuzzy preference conditions involved are of a bipolar nature (the first pole corresponding to a constraint, the second to a wish). More precisely, we consider queries where an inclusion-based condition comes into play (a typical example being a division query). Due to the fact that bipolar fuzzy queries entail using a framework where bipolar fuzzy relations are handled, we are led to defining generalized inclusion indicators in this context. This paper proposes an interpretation of such indicators and investigates their properties as well as their practical use in a database querying context.
\end{abstract}

Keywords: Database preference queries, bipolarity, inclusion, fuzzy relations.

\section{Introduction}

The idea of introducing preferences into queries is gaining more and more attention in the database community. In this paper, we focus on the fuzzyset-based approach to preference queries, which relies on the use of fuzzy set membership functions that describe the preference profiles of the user on each attribute domain involved in the query. Then, satisfaction degrees associated with elementary conditions may be combined using a panoply of fuzzy set connectives, which go much beyond conjunction and disjunction.

A complementary concept is that of bipolarity in general and its application to database querying. Bipolarity refers to the propensity of the human mind to reason and make decisions on the basis of positive and negative affects $[13,14]$. Positive information states what is possible, satisfactory, permitted, desired, or considered as being acceptable. On the other hand, negative statements express what is impossible, rejected, or forbidden. Negative preferences correspond to constraints, since they specify which values or objects have to be rejected (i.e., those that do not satisfy the constraints), while positive preferences correspond to wishes, as they specify which objects are more desirable than others (i.e., satisfy user wishes) without rejecting those that do not meet the wishes.
Three types of bipolarity have been pointed out [14]. The simplest type, called symmetric univariate bipolarity, uses a bipolar scale whose negative and positive parts are the mirror images of each other. The second type of bipolarity, termed symmetric bivariate, refers to the use of two separate unipolar scales (one for the positive affects, the other one for the negative affects) still pertaining to the same information, with generally a duality relation putting the scales in symmetric correspondence. The third type of bipolarity, called asymmetric, takes place when dealing with two unrelated kinds of information in parallel (see also [3] where this type of bipolarity is described and used). In the rest of this paper, asymmetric (also called heterogeneous) bipolarity is considered, and positive and negative poles are assumed to refer to potentially different notions (attributes). More precisely, we will deal with queries made of two poles, one meant as a constraint - denoted by $C$ - and the other acting as a wish - denoted by $W$-, and a pair $(C, W)$ is interpreted as: " $C$ and if possible $W$ ". In the situation considered later on, the two components of a query, although they can be assessed on a same scale (true/false, or the unit interval, or a qualitative scale), are not of the same nature and it is convenient to specify how any pair of elements (tuples or objects) are compared depending on their scores with respect to the constraint and the wish. Let us recall, however, that inside a complex constraint (resp. wish), the fuzzy-set-based approach requires the elementary preferences to be commensurable. A commonly made choice (see in particular [14]) for interpreting a bipolar condition consists in discriminating between two objects $x$ and $y$ using first the constraint, then if needed (i.e., if $x$ and $y$ are not distinguishable on the constraint) using the wish. In what follows, this point of view is chosen and a lexicographic order is used. If $(C(x), W(x))$ and $(C(y), W(y))$ denote the scores of $x$ and $y$ with respect to the constraint $C$ and the wish $W$, one has:

$$
\begin{aligned}
x \succ y \Leftrightarrow & (C(x)>C(y)) \text { or } \\
(C(x) & =C(y) \text { and } W(x)>W(y))
\end{aligned}
$$

where $x \succ y$ means that $x$ is preferred to $y$. A consequence is the fact that an object which is beaten on the constraint cannot win even if it is significantly better on the wish. 
In previous works, we have defined a fuzzy bipolar relational algebra [7], as well as a bipolar version of the fuzzy query language SQLf [20]. In this paper, our aim is to extend this latter work by considering new types of queries, namely those involving inclusion-based conditions. Taking into account bipolar fuzzy queries leads to handling bipolar fuzzy relations (i.e., relations where each tuple is assigned a pair of degrees, one related to the constraint, the other to the wish), thus the main issue is to give a semantics to the notion of inclusion between such bipolar fuzzy relations.

The remainder of the paper is structured as follows. Section 2 recalls basic notions related to the concept of bipolarity and to graded inclusion indicators based on fuzzy implications. Section 3 presents an interpretation of graded inclusion of fuzzy bipolar relations. The properties of such extended inclusion indicators are studied in Section 4. Section 5 is devoted to database preference queries involving inclusion-based conditions in the context where fuzzy bipolar relations have to be handled. Section 6 recalls the main contributions of the paper and outlines a few perspectives for future research.

\section{Preliminary notions}

In the following, we assume that satisfaction degrees belong to an ordinal symbolic scale $\mathcal{L}$ made of $k+1$ linguistic labels. For instance, with $k=4$, one may use:

$$
\begin{aligned}
& \omega_{0}\left(\text { "not at all") }<\omega_{1} \text { ("poorly") }<\omega_{2}\right. \text { ("medium") } \\
& <\omega_{3}\left(\text { "rather") }<\omega_{4}\right. \text { ("totally") }
\end{aligned}
$$

where $\omega_{0}$ (resp. $\omega_{k}$ ) corresponds to 0 (resp. 1) in the unit interval when a numeric framework is used. The operation $1-(\cdot)$ that is used to interpret the negation when the degrees belong to the unit interval is replaced by the order reversal operation denoted by $\operatorname{rev}(\cdot): \operatorname{rev}\left(\omega_{i}\right)=\omega_{k-i}$.

\subsection{Bipolarity}

In the following, we denote by $\mu(u)$ (resp. $\eta(u)$ ) the degree reflecting the extent to which an element $u$ satisfies a constraint $C$ (resp. a wish $W$ ). When $C$ and $W$ concern the same property of a given type of object or, in other terms, the same attribute of a given relation, two consistency conditions may be considered [14]:

- Strong consistency, as for twofold fuzzy sets [12]:

$$
\sup _{u} \min (\eta(u), \operatorname{rev}(\mu(u)))=\omega_{0}
$$

which expresses that the support of the wish must be included in the core of the constraint. Then the wish can only discriminate between the tuples which get degree $\omega_{k}$ for the constraint. As noted in [14], the pair $(W, C)$ under condition (2) is a twofold fuzzy set since $\operatorname{support}(W) \subseteq \operatorname{core}(C)$.

- Weak consistency (implied by the strong one), in the spirit of intuitionistic fuzzy sets [1]:

$$
\forall u, \eta(u) \leq \mu(u)
$$

which expresses that the wish must be included in the constraint (in the sense of Zadeh). Then, the wish can be used to discriminate between the tuples which somewhat satisfy the constraint. As noted in [14], the pair $(W, \bar{C})$ under condition (3) is an intuitionistic fuzzy set in the sense of Atanassov [1].

When $C$ and $W$ concern different attributes, the weak consistency of the wish with the constraint can be recovered by replacing $(C, W)$ by $(C, C \wedge W)$ as suggested in [14].

In the remainder of the paper, only weak consistency is enforced. We define a bipolar relation (in the database sense) as a relation where each tuple $t$ is associated with two degrees $\mu(t)$ and $\eta(t)$ in $\mathcal{L}$ expressing the extent to which the tuple satisfies the constraint (resp. wish) that has been used to produce the relation. In this framework, a tuple will then be denoted by $(\mu, \eta) / t$. In base relations (i.e., classical relations to which no fuzzy criterion has been applied yet), $\mu(t)=\eta(t)=\omega_{k}, \forall t$. It is assumed that tuples such that $\mu=\omega_{0}$ do not appear in the relation (they do not belong to it at all). Let us denote by $r$ a bipolar base relation and by $(C, W)$ the pair constraint/wish that is applied to $r$ in order to build a bipolar fuzzy relation $r^{\prime}$. One has:

$$
\begin{gathered}
r^{\prime}=\left\{(\mu, \eta) / t \mid\left(\omega_{k}, \omega_{k}\right) / t \in r \wedge \mu=\mu_{C}(t)\right. \\
\left.\wedge \eta=\eta_{W}(t)\right\} .
\end{gathered}
$$

When no constraint is expressed, Dubois and Prade [14] suggest to use $C=$ true. Notice that this case is not likely to be very useful in practice, since it corresponds to the situation where all the tuples from the relation queried are considered equally acceptable. The wish just allows to give a "bonus" to some items; for example, "I prefer red cars, but if there are not any, any car will do". Reciprocally, when no wish is expressed (case of a non-bipolar - fuzzy or not - condition), we use $W=C$. In this case, the condition acts as a regular select clause, i.e., it discards the items which do not satisfy the constraint at all (and ranks the others if the constraint is fuzzy).

We introduce the following operators $\operatorname{lmin}$ and lmax, which will play a major role for defining the conjunction (and intersection) and the disjunction (and union):

$$
\begin{aligned}
\operatorname{lmin} & \left((\mu, \eta),\left(\mu^{\prime}, \eta^{\prime}\right)\right) \\
& =(\mu, \eta) \text { if } \mu<\mu^{\prime} \text { or }\left(\mu=\mu^{\prime} \text { and } \eta<\eta^{\prime}\right) \\
& =\left(\mu^{\prime}, \eta^{\prime}\right) \text { otherwise. }
\end{aligned}
$$




$$
\begin{aligned}
\operatorname{lmax} & \left.(\mu, \eta),\left(\mu^{\prime}, \eta^{\prime}\right)\right) \\
& =(\mu, \eta) \text { if } \mu>\mu^{\prime} \text { or }\left(\mu=\mu^{\prime} \text { and } \eta>\eta^{\prime}\right) \\
& =\left(\mu^{\prime}, \eta^{\prime}\right) \text { otherwise. }
\end{aligned}
$$

These definitions may be reformulated the following way. Let us consider the scale $\mathcal{S}=\{(c, w) \mid c \in$ $\mathcal{L} \wedge w \in \mathcal{L} \wedge w \leq c\}$, equipped with the standard lexicographic order $\leq_{\text {lex }}$. Then the lmin and lmax operators are the canonical lattice operations of $\left(\mathcal{S}, \leq_{\text {lex }}\right)$ :

$$
\begin{aligned}
(c, w) & \leq_{\text {lex }}\left(c^{\prime}, w^{\prime}\right) \\
& \Leftrightarrow \operatorname{lmin}\left((c, w),\left(c^{\prime}, w^{\prime}\right)\right)=(c, w) \\
& \Leftrightarrow \operatorname{lmax}\left((c, w),\left(c^{\prime}, w^{\prime}\right)\right)=\left(c^{\prime}, w^{\prime}\right) .
\end{aligned}
$$

Straightforwardly, $\operatorname{lmin}$ (resp. lmax) is commutative, associative, idempotent, monotonic, has $\left(\omega_{k}\right.$, $\left.\omega_{k}\right)$ (resp. $\left.\left(\omega_{0}, \omega_{0}\right)\right)$ as a neutral element, and $\left(\omega_{0}, \omega_{0}\right)$ (resp. $\left.\left(\omega_{k}, \omega_{k}\right)\right)$ as an absorbing element. These properties make it legitimate to use $l$ min and lmax as conjunction and disjunction operators respectively (in the spirit of triangular norms and conorms).

Notice that Formula (4) can also be written:

$$
\begin{aligned}
r^{\prime} & =\{(\mu, \eta) / t \mid(\mu, \eta) \\
& \left.=\operatorname{lmin}\left(\left(\mu_{C}(t), \eta_{W}(t)\right),\left(\mu_{r}(t), \eta_{r}(t)\right)\right)\right\}
\end{aligned}
$$

since $r$ is a base relation (where $\mu_{r}(t)=\eta_{r}(t)=\omega_{k}$, $\forall t)$.

Note that relying on a lexicographic approach would make the approach questionable for queries involving a continuous attribute scale. Indeed, if one uses all of $[0,1]$, the following holds: $\forall \epsilon,(a, a)>_{\text {lex }}$ $(a, 0)>_{\text {lex }}(a-\epsilon, a-\epsilon)$, however small $\epsilon$ is. This might sound counterintuitive to a user. It makes less problems if $[0,1]$ is replaced by a small set of qualitative levels, and this is why we introduced the scale $\mathcal{L}$. See for instance [10] on qualitative vs. quantitative scales.

Remark 1. Even though the pairs of degrees we use resemble those handled in Atanassov's intuitionistic fuzzy sets, this resemblance is both natural and fallacious. On the one hand, Atanassov starts with genuine bipolar pairs $(p, n)=($ positive, negative) but interprets them as representing partially known membership grades $p \leq \mu \leq 1-n$, better captured by interval-valued fuzzy sets [11]. We rather use pairs $(l, u)=$ (wishes, constraints) where $l \leq u$, that in the literature are interpreted as uncertainty intervals, but that we interpret as expressing bipolarity. We could use instead pairs $(p, n)=($ wishes, rejections $)=(l, 1-u)$. In any case, the query is bipolar because the user expresses what he rejects (via constraints) and what he prefers ("I would like this but certainly not that"). Moreover, the bipolar semantics we consider leads us to a very different calculus from the one Atanassov advocates, more in line with his positive/negative starting point, while his calculus is not faithful to his original bipolar semantics, see [15].

\subsection{Graded inclusion based on a fuzzy implication}

A usual definition of the inclusion of $A$ in $B$ relies on the expression:

$$
(A \subseteq B) \Leftrightarrow(\forall x \in X,(x \in A) \Rightarrow(x \in B))
$$

which shows the tight relationship between inclusion and implication. This definition can also be written in terms of a constraint on the characteristic functions of $A$ and $B$ as:

$$
(A \subseteq B) \Leftrightarrow\left(\forall x \in X, f_{A}(x) \leq f_{B}(x)\right)
$$

This latter expression extends in a canonical way to two fuzzy sets $E$ and $F$ by:

$$
(E \subseteq F) \Leftrightarrow\left(\forall x \in X, \mu_{E}(x) \leq \mu_{F}(x)\right),
$$

which is often called "inclusion in the sense of Zadeh".

The major drawback of such definitions resides in the fact that a negative answer is obtained when the inclusion of $E$ in $F$ (regular or fuzzy sets) is "obviously" violated, but also when it almost holds. More generally, this phenomenon may be interpreted as the inability to distinguish between quite different situations, which is obviated using a graded inclusion.

Example 1. The fuzzy sets $E_{1}=\{1 / a, 1 / b\}$ and $E_{2}=\{0.5 / a, 0.7 / b\}$ are not included in the fuzzy set $F=\{0.4 / a, 0.7 / b\}$ using Formula (8). However, intuition may lead to consider that it is definitely more obvious for $E_{1}$ than for $E_{2}$ which is "almost

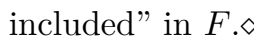

Devising an inclusion whose result is a degree in $[0,1]$ allows for a finer accounting than with an "all or nothing" view. The logical interpretation of graded inclusion [2] is based on the following extension of Formula (6) to fuzzy sets:

$$
\operatorname{deg}(E \subseteq F)=\min _{x \in X}\left(\mu_{E}(x) \rightarrow_{f} \mu_{F}(x)\right)
$$

$\rightarrow_{f}$ being a fuzzy implication.

It turns out that the nature of the inclusion obtained differs depending on the type of fuzzy implication used in Formula (9). In particular, if $E$ is included in $F$ according to Zadeh (8), the degree of inclusion is 1 for any R-implication (or its contraposition), but it is less than 1 when an S-implication is used. For instance, with Kleene-Dienes' or Reichenbach's implication, degree 1 is obtained iff the support of $E$ is included in the core of $F$.

Example 2. Let us consider the following fuzzy sets:

$E_{1}=\{0.4 / a, 0.3 / b\}$,

$E_{2}=\{1 / a, 0.9 / c\}$,

$F=\{1 / a, 0.8 / c\}$. 
According to Formula (9), for $\mathrm{i}=1$ or 2 , we have:

$$
\begin{aligned}
\operatorname{deg}\left(E_{i} \subseteq F\right)=\min ( & \mu_{E_{i}}(a) \rightarrow_{f} \mu_{F}(a), \\
\mu_{E_{i}}(b) & \rightarrow_{f} \mu_{F}(b), \\
\mu_{E_{i}}(c) & \left.\rightarrow_{f} \mu_{F}(c)\right),
\end{aligned}
$$

which, for Kleene-Dienes' implication leads to:

$$
\begin{aligned}
& \operatorname{deg}\left(E_{1} \subseteq F\right)= \\
& \quad \min \left(0.4 \rightarrow_{K} 1,0.3 \rightarrow_{K} 0,0 \rightarrow_{K} 0.8\right)=0.7, \\
& \operatorname{deg}\left(E_{2} \subseteq F\right) \\
& \quad=\min \left(1 \rightarrow_{K} 1,0 \rightarrow_{K} 0,0.9 \rightarrow_{K} 0.8\right)=0.8
\end{aligned}
$$

while using Gödel's implication, one gets:

$$
\begin{aligned}
& \operatorname{deg}\left(E_{1} \subseteq F\right)= \\
& \quad \min \left(0.4 \rightarrow_{G} 1,0.3 \rightarrow_{G} 0,0 \rightarrow_{G} 0.8\right)=0, \\
& \quad \operatorname{deg}\left(E_{2} \subseteq F\right) \\
& \quad=\min \left(1 \rightarrow_{G} 1,0 \rightarrow_{G} 0,0.9 \rightarrow_{G} 0.8\right)=0.8 . \diamond
\end{aligned}
$$

\section{Graded inclusion of bipolar fuzzy relations}

We start with Formula (9) and extend it to the case where the arguments of the fuzzy implication are pairs of degrees $(\mu, \eta)$.

$$
\begin{array}{r}
\operatorname{deg}(E \subseteq F)=\operatorname{lmin}_{x \in X}\left(\left(\mu_{E}(x), \eta_{E}(x)\right)\right. \\
\left.\rightarrow_{b f}\left(\mu_{F}(x), \eta_{F}(x)\right)\right)
\end{array}
$$

$\rightarrow_{b f}$ being a "bipolar fuzzy implication", i.e., a fuzzy implication extended to the case where its arguments are pairs of degrees. Notice that the choice is limited since the underlying fuzzy implication must work with a qualitative scale. One can use the two extended fuzzy implications $\rightarrow_{b G}$ and $\rightarrow_{b K}$ which are the counterparts of Gödel and KleeneDienes fuzzy implications respectively:

$$
\begin{aligned}
& \left(\mu_{1}, \eta_{1}\right) \rightarrow_{b G}\left(\mu_{2}, \eta_{2}\right) \\
& =\left\{\begin{array}{l}
\left(\omega_{k}, \omega_{k}\right) \text { if }\left(\mu_{1}, \eta_{1}\right) \leq_{\text {lex }}\left(\mu_{2}, \eta_{2}\right), \\
\left(\mu_{2}, \eta_{2}\right) \text { otherwise. }
\end{array}\right. \\
& \left(\mu_{1}, \eta_{1}\right) \rightarrow_{b K}\left(\mu_{2}, \eta_{2}\right) \\
& =\operatorname{lmax}\left(\neg\left(\mu_{1}, \eta_{1}\right),\left(\mu_{2}, \eta_{2}\right)\right) .
\end{aligned}
$$

Formula (12) raises the question of the interpretation of the negation $(\neg)$ of a bipolar condition. Several definitions have been proposed, cf. [6, 18, 7]. Let us consider for instance the definition from [18], which is recalled hereafter.

In [18], the negation of the fuzzy bipolar condition $(C, W)$ - meaning " $C$ and if possible $W$ "is defined as a new type of fuzzy bipolar condition denoted by $[\neg C, \neg W]$ where $[E, F]$ means " $E$ or else $F$ ". In this case, the positive pole $E$ specifies perfect values and the negative pole $F$ specifies acceptable ones (any element that does not satisfy $F$ is discarded). Reciprocally, the negation of the fuzzy bipolar condition $[E, F]$ is the fuzzy bipolar condition $(\neg E, \neg F)$.
With this definition of the negation, Formula (12) becomes:

$$
\begin{aligned}
& \left(\mu_{1}, \eta_{1}\right) \rightarrow_{b K}\left(\mu_{2}, \eta_{2}\right) \\
& \quad=\operatorname{lmax}\left(\left(\operatorname{rev}\left(\mu_{1}\right), \operatorname{rev}\left(\eta_{1}\right)\right),\left(\mu_{2}, \eta_{2}\right)\right) .
\end{aligned}
$$

The following example compares the results produced by Formula (10) when $\rightarrow_{b f}$ is $\rightarrow_{b G}$ as defined in Formula (11) or $\rightarrow_{b K}$ as defined in Formula (12).

Example 3. Let us consider a qualitative scale made of 7 levels $(k=6)$ :

$$
\omega_{0}<\omega_{1}<\ldots<\omega_{5}<\omega_{6}
$$

and the following bipolar fuzzy relations:

$E_{1}=\left\{\left(\omega_{3}, \omega_{3}\right) / a,\left(\omega_{2}, \omega_{0}\right) / b\right\}$,

$E_{2}=\left\{\left(\omega_{6}, \omega_{3}\right) / a,\left(\omega_{3}, \omega_{2}\right) / c\right\}$,

$F=\left\{\left(\omega_{3}, \omega_{2}\right) / a,\left(\omega_{5}, \omega_{4}\right) / c\right\}$.

According to Formula (10), for $\mathrm{i}=1$ or 2 , we have:

$$
\begin{aligned}
\operatorname{deg}\left(E_{i} \subseteq F\right) & \\
=\operatorname{lmin} & \left(\mu_{E_{i}}(a), \eta_{E_{i}}(a)\right) \rightarrow_{b f}\left(\mu_{F}(a), \eta_{F}(a)\right), \\
& \left(\mu_{E_{i}}(b), \eta_{E_{i}}(b)\right) \rightarrow_{b f}\left(\mu_{F}(b), \eta_{F}(b)\right), \\
& \left.\left(\mu_{E_{i}}(c), \eta_{E_{i}}(c)\right) \rightarrow_{b f}\left(\mu_{F}(c), \eta_{F}(c)\right)\right),
\end{aligned}
$$

which, using the extension of Gödel' implication (Formula (11)) leads to:

$$
\begin{gathered}
\operatorname{deg}\left(E_{1} \subseteq F\right)=\operatorname{lmin}\left(\left(\omega_{3}, \omega_{3}\right) \rightarrow_{b G}\left(\omega_{3}, \omega_{2}\right),\right. \\
\left(\omega_{2}, \omega_{0}\right) \rightarrow_{b G}\left(\omega_{0}, \omega_{0}\right), \\
\left.\left(\omega_{0}, \omega_{0}\right) \rightarrow_{b G}\left(\omega_{5}, \omega_{4}\right)\right) \\
=\operatorname{lmin}\left(\left(\omega_{3}, \omega_{2}\right),\left(\omega_{0}, \omega_{0}\right),\left(\omega_{6}, \omega_{6}\right)\right)=\left(\omega_{0}, \omega_{0}\right), \\
\operatorname{deg}\left(E_{2} \subseteq F\right)=\operatorname{lmin}\left(\left(\omega_{6}, \omega_{3}\right) \rightarrow_{b G}\left(\omega_{3}, \omega_{2}\right),\right. \\
\left.\quad\left(\omega_{0}, \omega_{0}\right) \rightarrow_{b G}\left(\omega_{0}, \omega_{0}\right)\right) \\
\left.\quad\left(\omega_{3}, \omega_{2}\right) \rightarrow_{b G}\left(\omega_{5}, \omega_{4}\right)\right) \\
=\operatorname{lmin}\left(\left(\omega_{3}, \omega_{2}\right),\left(\omega_{6}, \omega_{6}\right),\left(\omega_{6}, \omega_{6}\right)\right) \\
=\left(\omega_{3}, \omega_{2}\right),
\end{gathered}
$$

while using the extension of Kleene-Dienes' implication (Formula (13)), one gets:

$$
\begin{gathered}
\operatorname{deg}\left(E_{1} \subseteq F\right)=\operatorname{lmin}\left(\left(\omega_{3}, \omega_{3}\right) \rightarrow_{b K}\left(\omega_{3}, \omega_{2}\right),\right. \\
\left(\omega_{2}, \omega_{0}\right) \rightarrow_{b K}\left(\omega_{0}, \omega_{0}\right) \\
\left.\left(\omega_{0}, \omega_{0}\right) \rightarrow_{b K}\left(\omega_{5}, \omega_{4}\right)\right) \\
=\operatorname{lmin}\left(\operatorname{lmax}\left(\left(\operatorname{rev}\left(\omega_{3}\right), \operatorname{rev}\left(\omega_{3}\right)\right),\left(\omega_{3}, \omega_{2}\right)\right),\right. \\
\operatorname{lmax}\left(\left(\operatorname{rev}\left(\omega_{2}\right), \operatorname{rev}\left(\omega_{0}\right)\right),\left(\omega_{0}, \omega_{0}\right)\right) \\
\left.\operatorname{lmax}\left(\left(\operatorname{rev}\left(\omega_{0}\right), \operatorname{rev}\left(\omega_{0}\right)\right),\left(\omega_{5}, \omega_{4}\right)\right)\right) \\
=\operatorname{lmin}\left(\left(\omega_{3}, \omega_{3}\right),\left(\omega_{4}, \omega_{6}\right),\left(\omega_{6}, \omega_{6}\right)\right) \\
\left.=\left(\omega_{3}, \omega_{3}\right), \quad\left(\omega_{0}, \omega_{0}\right) \rightarrow_{b K}\left(\omega_{0}, \omega_{0}\right)\right) \\
\operatorname{deg}\left(E_{2} \subseteq F\right)=\operatorname{lmin}\left(\left(\omega_{6}, \omega_{3}\right) \rightarrow_{b K}\left(\omega_{3}, \omega_{2}\right),\right. \\
=\operatorname{lmin}\left(\operatorname{lmax}\left(\left(\operatorname{rev}\left(\omega_{6}\right), \operatorname{rev}\left(\omega_{3}\right)\right),\left(\omega_{3}, \omega_{2}\right)\right),\right. \\
\quad \operatorname{lmax}\left(\left(\operatorname{rev}\left(\omega_{0}\right), \operatorname{rev}\left(\omega_{0}\right)\right),\left(\omega_{0}, \omega_{0}\right)\right) \\
\left.\operatorname{lmax}\left(\left(\operatorname{rev}\left(\omega_{3}\right), \operatorname{rev}\left(\omega_{2}\right)\right),\left(\omega_{5}, \omega_{4}\right)\right)\right) \\
=\operatorname{lmin}\left(\left(\omega_{3}, \omega_{2}\right),\left(\omega_{6}, \omega_{6}\right),\left(\omega_{5}, \omega_{4}\right)\right) \\
=\left(\omega_{3}, \omega_{2}\right) . \diamond \quad
\end{gathered}
$$

As in the fuzzy unipolar case, the differences between the results obtained with Gödel and KleeneDienes implications come from the fact that in the 
first case, the left-hand side acts as a threshold (behavior of an R-implication) whereas it expresses importance in the second case.

Anyway, one may wonder whether Formula (10) is the most suitable, considering the fact that the interpretation of its result, i.e. a pair of degrees $(\mu, \eta)$, is somewhat at odds with the interpretation of the pairs of degrees associated with the elements in the relations handled. Indeed, in these relations, a pair of degrees reflects the satisfaction of a bipolar event according to the semantics "and if possible" or "or else" in case of a negated bipolar condition -, which is not the case here (the event that is assessed, i.e. the inclusion of $E$ in $F$, is not bipolar, only the arguments are).

A solution to this semantic issue is to view the inclusion of bipolar relations as an event that is bipolar itself. A possible choice is to consider the event "the desirable elements of $E$ are included in the acceptable elements of $F$ (constraint), and even better, the acceptable elements of $E$ are included in the desirable elements of $F$ (wish)". In this statement "acceptable" refers to the satisfaction of the constraint (degree $\mu$ ) underlying the bipolar relation whereas "desirable" refers to the satisfaction of the wish (degree $\eta$ ). This leads to defining:

$$
\operatorname{deg}(E \subseteq F)=\left(\mu_{1}, \eta_{1}\right)
$$

where

$$
\begin{array}{r}
\mu_{1}=\operatorname{deg}(\text { desirable }(E) \subseteq \operatorname{acceptable}(F)) \\
=\min _{x \in X} \eta_{E}(x) \rightarrow_{f} \mu_{F}(x)
\end{array}
$$

and

$$
\begin{array}{r}
\eta_{1}=\operatorname{deg}(\operatorname{acceptable}(E) \subseteq \text { desirable }(F)) \\
=\min _{x \in X} \mu_{E}(x) \rightarrow_{f} \eta_{F}(x) .
\end{array}
$$

Notice that Formula (14) corresponds to a rather lax view of bipolar inclusion inasmuch as $\mu_{1}$ may be equal to $\omega_{k}$ even when many elements that are totally in acceptable $(E)$ are completely outside acceptable $(F)$. An alternative definition of bipolar inclusion could be based on the bipolar event "the acceptable elements of $E$ are included in the acceptable elements of $F$ (constraint), and even better, the desirable elements of $E$ are included in the desirable elements of $F$ (wish)". But then, the wish would not constitute a refinement (strengthening) of the constraint as it is the case with Formula (14). On the other hand, a more satisfactory alternative is to consider the bipolar event "the acceptable elements of $E$ are included in the acceptable elements of $F$ (constraint), and even better, the acceptable elements of $E$ are included in the desirable elements of $F$ (wish)". This leads to the alternative definition (where $\eta_{E}$ does not play any role anymore):

$$
\operatorname{deg}(E \subseteq F)=\left(\mu_{2}, \eta_{1}\right)
$$

where

$$
\begin{array}{r}
\mu_{2}=\operatorname{deg}(\operatorname{acceptable}(E) \subseteq \operatorname{acceptable}(F)) \\
=\min _{x \in X} \mu_{E}(x) \rightarrow_{f} \mu_{F}(x)
\end{array}
$$

and $\eta_{1}$ is defined as in Formula (16) above.

Example 4. Let us consider again the fuzzy bipolar relations from Example 3, as well as a qualitative scale made of 7 levels. Let us now use Formula (14) to assess the inclusion $E \subseteq F$. With Gödel's implication, we get for $E_{1}$ :

$$
\begin{aligned}
\mu_{1} & =\min \left(\omega_{3} \rightarrow_{G} \omega_{3}, \omega_{0} \rightarrow_{G} \omega_{0}, \omega_{0} \rightarrow_{G} \omega_{5}\right) \\
& =\min \left(\omega_{6}, \omega_{6}, \omega_{6}\right)=\omega_{6} . \\
\eta_{1} & =\min \left(\omega_{3} \rightarrow_{G} \omega_{2}, \omega_{2} \rightarrow_{G} \omega_{0}, \omega_{0} \rightarrow_{G} \omega_{4}\right) \\
& =\min \left(\omega_{2}, \omega_{0}, \omega_{6}\right)=\omega_{0} .
\end{aligned}
$$

Thus: $\operatorname{deg}\left(E_{1} \subseteq_{G} F\right)=\left(\omega_{6}, \omega_{0}\right)$.

With Kleene-Dienes' implication, we get:

$$
\begin{aligned}
\mu_{1} & =\min \left(\omega_{3} \rightarrow_{K} \omega_{3}, \omega_{0} \rightarrow_{K} \omega_{0}, \omega_{0} \rightarrow_{K} \omega_{5}\right) \\
& =\min \left(\omega_{3}, \omega_{6}, \omega_{6}\right)=\omega_{3} . \\
\eta_{1} & =\min \left(\omega_{3} \rightarrow_{K} \omega_{2}, \omega_{2} \rightarrow_{K} \omega_{0}, \omega_{0} \rightarrow_{K} \omega_{4}\right) \\
& =\min \left(\omega_{3}, \omega_{4}, \omega_{6}\right)=\omega_{3} .
\end{aligned}
$$

Thus: $\operatorname{deg}\left(E_{1} \subseteq_{K} F\right)=\left(\omega_{3}, \omega_{3}\right)$.

Similarly, for $E_{2}$, we get:

$\operatorname{deg}\left(E_{2} \subseteq_{G} F\right)=\left(\omega_{6}, \omega_{2}\right)$ and

$\operatorname{deg}\left(E_{2} \subseteq_{K} F\right)=\left(\omega_{3}, \omega_{2}\right)$.

Using Formula (17), we get for $E_{1}$ :

$\operatorname{deg}\left(E_{1} \subseteq_{G} F\right)=\left(\omega_{0}, \omega_{0}\right)$ and $\operatorname{deg}\left(E_{1} \subseteq_{K} F\right)=\left(\omega_{3}, \omega_{3}\right)$.

and for $E_{2}$ :

$\operatorname{deg}\left(E_{2} \subseteq_{G} F\right)=\left(\omega_{3}, \omega_{2}\right)$ and $\operatorname{deg}\left(E_{2} \subseteq_{K} F\right)=\left(\omega_{3}, \omega_{2}\right) . \diamond$

\section{Properties}

Several researchers axiomatized the graded inclusion of fuzzy sets. Sinha and Dougherty [19], in particular, defined the following set of axioms. Let $X$ be a universe and $F(X)$ the class of the fuzzy sets defined over $X$.

- (S1) $\operatorname{Inc}(A, B)=1 \Leftrightarrow A \subseteq B$ in Zadeh's sense;

- (S2) $\operatorname{Inc}(A, B)=0 \Leftrightarrow \exists x \in X$ such that $A(x)=1$ and $B(x)=0$;

- (S3) Inc has increasing second partial mapping: $B \subseteq C \Rightarrow \operatorname{Inc}(A, B) \leq \operatorname{Inc}(A, C)$;

- (S4) Inc has decreasing first partial mapping: $B \subseteq C \Rightarrow \operatorname{Inc}(C, A) \leq \operatorname{Inc}(B, A)$;

- (S5) $\operatorname{Inc}(A, B)=\operatorname{Inc}(S(A), S(B))$ where $S$ is a $F(X) \rightarrow F(X)$ mapping defined by: $\forall x \in$ $X, S(A)(x)=A(s(x)), s$ denoting an $X \rightarrow X$ mapping.

- (S6) $\operatorname{Inc}(A, B)=\operatorname{Inc}\left(B^{C}, A^{C}\right)$ where $A^{C}$ (resp. $B^{C}$ ) denotes the complement of $A$ (resp. $B)$ in the universe $X$;

- (S7) $\operatorname{Inc}(A \cup B, C)=\min (\operatorname{Inc}(A, C), \operatorname{Inc}(B$, $C)$ ) where $\cup$ is interpreted by max; 
- (S8) $\operatorname{Inc}(A, B \cap C)=\min (\operatorname{Inc}(A, B), \operatorname{Inc}(A$,

$C)$ ) where $\cap$ is interpreted by min.

Independently of Sinha and Dougherty, Kitainik [17] developed an axiomatic approach to the treatment of fuzzy inclusion indicators. Kitainik's requirements are given hereafter:

- (K1) $\operatorname{Inc}(A, B)=\operatorname{Inc}\left(B^{C}, A^{C}\right)$;

- $(\mathrm{K} 2) \operatorname{Inc}(A, B \cap C)=\min (\operatorname{Inc}(A, B), \operatorname{Inc}(A$, $C)$ );

- (K3) $\operatorname{Inc}(A, B)=\operatorname{Inc}(S(A), S(B))$ where $S$ is defined as in S5 above;

- (K4) When applied to crisp sets, Inc coincides with crisp set inclusion.

Fodor and Yager [16] showed that the admissible (in the sense of Kitainik's requirements) inclusion indicators belong to the Bandler-Kohout class [2] (i.e., the inclusion indicators defined by Formula (9) above) and are based on a contrapositive fuzzy implication, i.e., a fuzzy implication $F$ such that $F(x, y)=F\left(N_{F}(y), N_{F}(x)\right) \forall x, y \in[0,1]$, with $N_{F}$ the induced negator of $F$ defined as $N_{F}(x)=$ $F(x, 0) \forall x \in[0,1]$. Kitainik proved that in the Sinha-Dougherty axiom list, Axioms S3, S4 and S7 are a direct consequence of Axioms S1, S2, S5, S6 and S8. On the other hand, Cornelis et al. [9] pointed out that the inclusion indicators which satisfy the axioms proposed by Sinha-Dougherty are a subclass of those satisfying Kitainik's requirements. Let us now check whether Kitainik's axioms remain valid in the context of bipolar fuzzy relations, i.e., when Formula (14) is used.

- $\left(\mathrm{K}^{\prime}\right) \operatorname{deg}(A \subseteq B)=\operatorname{deg}\left(B^{C} \subseteq A^{C}\right)$ does not hold with the definition of the negation from [18]. For this axiom to be valid, one would have to have:

$$
\begin{aligned}
& \eta_{A}(x) \rightarrow_{f} \mu_{B}(x)= \\
& \quad \operatorname{rev}\left(\eta_{B}(x)\right) \rightarrow_{f} \operatorname{rev}\left(\mu_{A}(x)\right) \\
& \mu_{A}(x) \rightarrow_{f} \eta_{B}(x)= \\
& \quad \operatorname{rev}\left(\mu_{B}(x)\right) \rightarrow_{f} \operatorname{rev}\left(\eta_{A}(x)\right),
\end{aligned}
$$

which is not true in general.

- $\left(\mathrm{K} 2^{\prime}\right)$ The property:

$$
\begin{aligned}
\operatorname{deg}(A \subseteq & B \cap C)= \\
& \operatorname{lmin}(\operatorname{deg}(A \subseteq B), \operatorname{deg}(A \subseteq C))
\end{aligned}
$$

holds due to the definition of $\operatorname{lmin}$ and the monotonicity of fuzzy implications. The complete proof is omitted due to space limit but its crux is that $\forall \mu_{A}, \eta_{A}, \mu_{B}, \eta_{B}, \mu_{C}, \eta_{C}$ :

$$
\begin{gathered}
\left(\eta_{A} \rightarrow \operatorname{lmin}\left(\left(\mu_{B}, \eta_{B}\right),\left(\mu_{C}, \eta_{C}\right)\right)[\mu],\right. \\
\left.\mu_{A} \rightarrow \operatorname{lmin}\left(\left(\mu_{B}, \eta_{B}\right),\left(\mu_{C}, \eta_{C}\right)\right)[\eta]\right) \\
=\operatorname{lmin}\left(\left(\eta_{A} \rightarrow \mu_{B}, \mu_{A} \rightarrow \eta_{B}\right),\right. \\
\left.\left(\eta_{A} \rightarrow \mu_{C}, \mu_{A} \rightarrow \eta_{C}\right)\right)
\end{gathered}
$$

where $[\mu]$ (resp. $[\eta]$ ) denotes the projection of a pair of degrees on its first (resp. second) component.
- $\left(\mathrm{K} 3^{\prime}\right) \operatorname{deg}(A \subseteq B)=\operatorname{deg}(S(A), S(B))$ where $S$ is defined as in Axiom S5: straightforwardly holds;

- $\left(\mathrm{K} 4^{\prime}\right)$ When applied to crisp sets, $\operatorname{deg}(A \subseteq B)$ coincides with crisp set inclusion: holds since i) in that case, $\mu_{A}(x)=\eta_{A}(x)$ and $\mu_{B}(x)=$ $\eta_{B}(x) \forall x$, and ii) fuzzy implications coincide with the classical (Boolean) implication when their arguments are Boolean.

Notice that the only axiom that does not hold, namely the contrapositivy axiom (K1), is very constraining - even in the unipolar case - since it dismisses all the fuzzy implications that are not contrapositive (which is the case of most R-implications, in particular). We do not think that this axiom should be considered mandatory in the database framework, considering that the complementation operation never intervenes at a query level in such a context.

Remark 2. The same three axioms are preserved if Formula (17) is used instead of Formula (14).

\section{Application to database querying}

The general framework is that of bipolar fuzzy relations, i.e., relations where each tuple $t$ is associated with two degrees $\mu(t)$ and $\eta(t)$ in $\mathcal{L}$ expressing the extent to which the tuple satisfies the constraint (resp. wish) that has been used to produce the relation (cf. Subsection 2.1).

A typical example of database queries involving an inclusion, dealt with hereafter, is division queries.

\subsection{Reminder about the division}

We assume that the dividend relation $r$ has the schema $(X, A)$, while that of the divisor relation $s$ is $(B)$ where $A$ and $B$ are compatible sets of attributes. The division of relation $r$ by relation $s$ may be defined as follows:

$$
r[A \div B] s=\left\{x \in \pi_{X}(r) \mid s \subseteq \Omega(x)\right\}
$$

where $\pi_{X}(r)$ denotes the projection of $r$ over $X$ and $\Omega(x)=\{a \mid\langle x, a\rangle \in r\}$. In other words, an element $x$ belongs to the result of the division of $r$ by $s$ iff it is associated in $r$ with at least all the values $a$ appearing in $s$.

Example 5. Let us consider a database involving the two relations order $(o)$ and product $(p)$ with respective schemas $O(n p$, store, qty) and $P(n p$, price). Tuples $(n, s, q)$ of $o$ and $(n, p r)$ of $p$ state that product $n$ has been ordered from store $s$ in quantity $q$ and that its price is $p r$. Retrieving the stores which have been ordered all the products priced under $\$ 127$ in a quantity greater than 35 , can be expressed thanks to a division as:

$$
o_{g 35}[n p \div n p] p_{u 127}
$$


where relation $o_{g 35}$ corresponds to pairs $(s, n)$ such that product $n$ has been ordered from store $s$ in a quantity over 35 and relation $p_{u 127}$ gathers products whose price is under $\$ 127$. With the extensions of relations $o_{g 35}$ and $p_{u 127}$ given hereafter:

$o_{g 35}=\left\{\left\langle s_{32}, p_{15}\right\rangle,\left\langle s_{32}, p_{12}\right\rangle,\left\langle s_{32}, p_{34}\right\rangle,\left\langle s_{32}, p_{26}\right\rangle\right.$, $\left.\left\langle s_{7}, p_{12}\right\rangle,\left\langle s_{7}, p_{26}\right\rangle,\left\langle s_{19}, p_{15}\right\rangle,\left\langle s_{19}, p_{12}\right\rangle,\left\langle s_{19}, p_{26}\right\rangle\right\}$ $p_{u 127}=\left\{\left\langle p_{15}\right\rangle,\left\langle p_{12}\right\rangle,\left\langle p_{26}\right\rangle\right\}$

the division returns $\left\{\left\langle s_{32}\right\rangle,\left\langle s_{19}\right\rangle\right\} . \diamond$

When the relations involved are fuzzy (i.e. contain graded tuples), Equation (19) may be interpreted as follows [8]:

$$
\begin{aligned}
& \mu_{r[A \div B] s}(x)= \\
& \inf _{a \in \operatorname{support}(s)} \mu_{s}(a) \rightarrow_{f} \mu_{r}(x, a)
\end{aligned}
$$

where $\rightarrow_{f}$ denotes a fuzzy implication.

Example 6. Let us consider again the relations from Example 5, a scale $\mathcal{L}$ such that $k=7$, and two fuzzy conditions "quantity is around 30 " and "price is around $\$ 100 "$ applied on $O$ and $P$ respectively, leading to two fuzzy relations $o_{a 30}$ and $p_{a 100}$ whose extensions are given hereafter:

$o_{a 30}=\left\{\omega_{4} /\left\langle s_{32}, p_{11}\right\rangle, \omega_{7} /\left\langle s_{32}, p_{17}\right\rangle, \omega_{5} /\left\langle s_{32}, p_{29}\right\rangle\right.$, $\omega_{6} /\left\langle s_{7}, p_{11}\right\rangle, \omega_{5} /\left\langle s_{7}, p_{29}\right\rangle, \omega_{7} /\left\langle s_{19}, p_{11}\right\rangle, \omega_{2} /\left\langle s_{19}\right.$, $\left.\left.p_{17}\right\rangle, \omega_{6} /\left\langle s_{19}, p_{42}\right\rangle\right\}$

$p_{a 100}=\left\{\omega_{7} /\left\langle p_{11}\right\rangle, \omega_{6} /\left\langle p_{17}\right\rangle, \omega_{5} /\left\langle p_{29}\right\rangle\right\}$.

The division of $o_{a 30}$ by $p_{a 100}$ based on Gödel's implication returns $\left\{\omega_{4} /\left\langle s_{32}\right\rangle, \omega_{2} /\left\langle s_{19}\right\rangle\right\} . \diamond$

\subsection{Division of bipolar fuzzy relations}

Let us now consider the case where $r$ and $s$ may be bipolar fuzzy relations. Starting with Formula (19), we define:

$$
\left(\mu_{r[A \div B] s}(x), \eta_{r[A \div B] s}(x)\right)=\operatorname{deg}(s \subseteq \Omega(x))
$$

where $\operatorname{deg}(s \subseteq \Omega(x))$ is interpreted according to Formula (14).

Let us denote by birel the operation - which is not part of the query language itself — that builds a bipolar fuzzy relation from two consistent (in the sense of weak consistency) regular fuzzy relations:

$$
\begin{aligned}
\operatorname{birel}(r, s)= & \\
\{(\mu, \eta) / t \mid t & \in \operatorname{support}(r) \wedge \mu_{r}(t)=\mu \wedge \\
t & \left.\in \operatorname{support}(s) \wedge \mu_{s}(t)=\eta\right\} \\
\cup\{(\mu, 0) / t \mid t & \in \operatorname{support}(r) \wedge \mu_{r}(t)=\mu \wedge \\
t & \notin \operatorname{support}(s)\} .
\end{aligned}
$$

where $\mu_{r}(t)$ (resp. $\left.\mu_{s}(t)\right)$ denotes the membership degree associated with tuple $t$ in the regular fuzzy relation $r$ (resp. $s$ ). The definition of the division of bipolar fuzzy relations can be rewritten as:

$$
r[A \div B] s=\operatorname{birel}\left(r_{C}[A \div B] s_{W}, r_{W}[A \div B] s_{C}\right)
$$

where rel $_{C}$ (resp. rel $_{W}$ ) denotes the fuzzy relation obtained by keeping only the degrees $\mu$ (resp. $\eta$ ) attached to the tuples from the bipolar fuzzy relation rel.
In practice, it is likely that either the divisor or the dividend will be bipolar but not both since the meaning of the query then becomes rather complex. Anyway, Formula (14) makes it possible to deal with the general case where both are.

Example 7. Let us consider again the relations $O$ and $P$ from Example 5. Let us now assume that a bipolar relation $O_{b}$ is built from $O$ using the bipolar condition:

\section{$\left(C_{O}:\right.$ qty is higher_than_10, \\ $W_{O}$ : qty is higher_than_15)}

where higher_than_10 and higher_than_15 are assumed to be fuzzy predicates. An example of a division query involving a bipolar dividend is: "find the stores from which at least 10 occurrences of each product have been ordered, and if possible at least 15 occurrences of each product. Here, the bipolar fuzzy dividend is $O_{b}$ whereas the divisor is $P$.

Now assume that a bipolar relation $P_{b}$ is built from $P$ using the bipolar condition:

$\left(C_{P}:\right.$ price is less_than_200,

$W_{P}$ : price is less_than_150)

where less_than_200 and less_than_150 are fuzzy predicates. An example of a division query involving a bipolar divisor is: "find the stores from which all of the products priced less than $\$ 200$ have been ordered, and if possible all of those priced less than $\$ 150 "$. In this case, the unipolar dividend is $O$ whereas the fuzzy bipolar divisor is $P_{b} . \diamond$

Let us mention that an alternative semantics for the bipolar division of unipolar fuzzy relations is studied in [5], where bipolarity is conveyed by a stratified divisor.

Remark 3. Considering the definition given above, processing a division $r[A \div B] s$ of two bipolar fuzzy relations $r$ and $s$ comes down to processing two divisions of non-bipolar fuzzy relations, namely $r_{C}[A \div B] s_{W}$ and $r_{W}[A \div B] s_{C}$ - cf. Equation (23). Since it has been proven that the result of the division of two fuzzy relations is a quotient provided that the conjunction operator used for the Cartesian product is appropriately chosen [8], the result of a division of bipolar fuzzy relations can be characterized as a twofold quotient. However, when the implication used is an S-implication, the quotient property can be guaranteed only if the divisor is normalized. In the context of bipolar relations, this means that both $s_{C}$ and $s_{W}$ have to contain a tuple whose associated degree equals 1.

Remark 4. Basically, the processing of a division of bipolar fuzzy relations obeys the same principle as in the unipolar case (see [4]). The only thing that changes is that it is necessary to process two divisions of unipolar fuzzy relations (cf. Formula (23)) instead of one, which means that the practical complexity is multiplied by two, but the class of data complexity stays the same. 


\section{Conclusion}

In this paper, we have studied how the notion of a graded inclusion could be extended to the case where the sets involved result from the evaluation of two bipolar fuzzy conditions on two regular sets. After considering several possible interpretations of such an extended inclusion, we focused on a view that consists in seeing inclusion as a bipolar event whose assessment is based on the inclusion of the desirable (resp. acceptable) elements of a set in the acceptable (resp. desirable) elements of the other. We have then studied the properties of the corresponding bipolar inclusion indicator, and showed how it could be applied in a context of database flexible querying.

Perspectives concern for instance implementation issues, and we plan on integrating the bipolar inclusion operator defined here into a fuzzy querying prototype named PostgreSQLf that we have developed over the open source RDBMS PostgreSQL. Another objective is to investigate other types of application (outside the database context) for this bipolar inclusion operator.

\section{References}

[1] K. Atanassov. Intuitionistic fuzzy sets. Fuzzy Sets and Systems, 20(1):87-96, 1986.

[2] W. Bandler and L. Kohout. Fuzzy power sets and fuzzy implication operators. Fuzzy Sets and Systems, 4:13-30, 1980.

[3] S. Benferhat, D. Dubois, S. Kaci, and H. Prade. Bipolar possibility theory in preference modeling: Representation, fusion and optimal solutions. Information Fusion, 7(1):135-150, 2006.

[4] P. Bosc, C. Legrand, and O. Pivert. About fuzzy query processing - The example of the division. In Proc. of the 8th IEEE International Conference on Fuzzy Systems (FUZZIEEE'99), pages 592-597, Seoul, Korea, 1999.

[5] P. Bosc and O. Pivert. On diverse approaches to bipolar division operators. Int. J. Intell. Syst., 26(10):911-929, 2011.

[6] P. Bosc and O. Pivert. On the negation of bipolar fuzzy conditions. In Proc. of the 30th International Conference of the North American Fuzzy Information Processing Society (NAFIPS'11), El Paso, TX, USA, 2011.

[7] P. Bosc and O. Pivert. On a fuzzy bipolar relational algebra. Inf. Sci., 219:1-16, 2013.

[8] P. Bosc, O. Pivert, and D. Rocacher. About quotient and division of crisp and fuzzy relations. Journal of Intelligent Information Systems, 29(2):185-210, 2007.

[9] C. Cornelis, C. Van der Donck, and E. E. Kerre. Sinha-dougherty approach to the fuzzification of set inclusion revisited. Fuzzy Sets and Systems, 134(2):283-295, 2003.
[10] D. Dubois. The role of fuzzy sets in decision sciences: Old techniques and new directions. Fuzzy Sets and Systems, 184(1):3-28, 2011.

[11] D. Dubois, S. Gottwald, P. Hájek, J. Kacprzyk, and H. Prade. Terminological difficulties in fuzzy set theory - The case of "intuitionistic fuzzy sets". Fuzzy Sets and Systems, 156(3):485-491, 2005.

[12] D. Dubois and H. Prade. Twofold fuzzy sets and rough sets - Some issues in knowledge representation. Fuzzy Sets and Systems, 23(1):3-18, 1987.

[13] D. Dubois and H. Prade. Bipolarity in flexible querying. In T. Andreasen, A. Motro, H. Christiansen, and H. L. Larsen, editors, FQAS, volume 2522 of Lecture Notes in Computer Science, pages 174-182. Springer, 2002.

[14] D. Dubois and H. Prade. Handling bipolar queries in fuzzy information processing. In J. Galindo, editor, Handbook of Research on Fuzzy Information Processing in Databases, pages 97-114. Information Science Reference, Hershey, PA, USA, 2008.

[15] D. Dubois and H. Prade. Gradualness, uncertainty and bipolarity: Making sense of fuzzy sets. Fuzzy Sets and Systems, 192:3-24, 2012.

[16] J. Fodor and R. Yager. Fuzzy-set theoretic operators and quantifiers. In D. Dubois and H. Prade, editors, The Handbooks of Fuzzy Sets Series, vol. 1: Fundamentals of Fuzzy Sets, pages 125-193. Kluwer Academic Publishers, Dordrecht, The Netherlands, 2000.

[17] L. Kitainik. Fuzzy implication and fuzzy inclusion: a comparative axiomatic study. In R. Lowen and M. Roubens, editors, Fuzzy Logic - State of the Art, pages 441-451. Kluwer Academic Publishers, Dordrecht, The Netherlands, 1993.

[18] L. Liétard, N. Tamani, and D. Rocacher. Fuzzy bipolar conditions of type "or else". In FUZZIEEE, pages 2546-2551. IEEE, 2011.

[19] D. Sinha and E. Dougherty. Fuzzification of set inclusion: theory and applications. Fuzzy Sets and Systems, 55:15-42, 1993.

[20] N. Tamani, L. Liétard, and D. Rocacher. Bipolar SQLf: A flexible querying language for relational databases. In H. Christiansen, G. D. Tré, A. Yazici, S. Zadrozny, T. Andreasen, and H. L. Larsen, editors, FQAS, volume 7022 of Lecture Notes in Computer Science, pages 472 484. Springer, 2011. 